\title{
NEW THEOREM ON PERPENDICULAR BISECTORS OF FOCAL RADII OF AN ELLIPSE
}

\author{
A.K. Maran*
}

\begin{abstract}
Ellipse' is one of the conic sections, it is sort of elongated circle. It is the locus of a point that moves in such a way that the ratio of its distance from a fixed point to its distance from a fixed line equals to constant ' $e$ '. According to Keplar's law of planetary motion, ${ }^{2}$ the ellipse is very important in the field of Astronomy. The author has developed a new theorem for a new property of ellipse.
\end{abstract}

Key words: Ellipse, Focal radius, Perpendicular bisector, Major axis and Minor axis

\section{New theorem on Ellipse}

"If two focal radii ${ }^{3}$ (pair) are drawn through both foci ${ }^{4}$ from a point anywhere on peripheral of an ellipse (except extremities of major axis), then the perpendicular bisectors ${ }^{5}$ of these two focal radii meets at line of minor axis. ${ }^{6}$."

* Animal Health Research Unit, St. Xavier's College, Paloyamkottai - 627002.

E-mail: repro_mannan@yahoo.co.in 


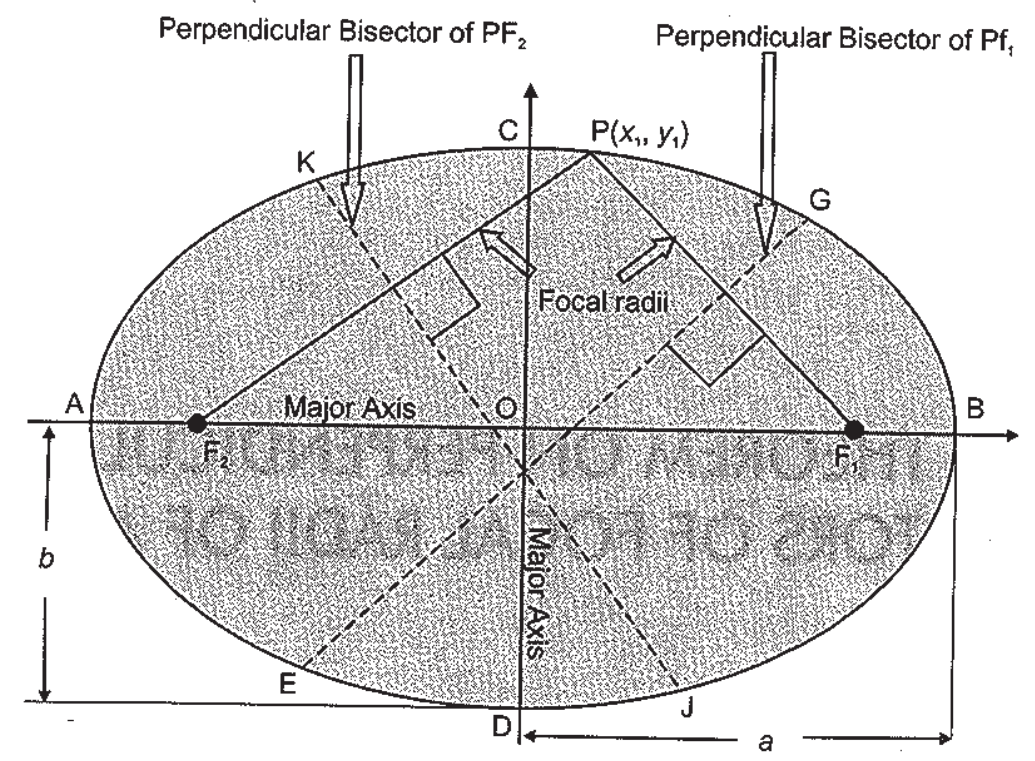

Figure 1 : Ellipse

Figurel is the typical diagram of an ellipse. In this figure, ${ }^{\prime} O$ ' is the centre of ellipse, $F_{1} \& F_{2}$ are the foci, $A, B \& C, D$ are the extremities of major and minor axis respectively, ' $P$ ' is the point anywhere on periphery of the ellipse (except extremities of major axis), $P F_{1} \& P F_{2}$ are the pair focal radii through $F_{1} \& F_{2}$ respectively. $E G$ \& $\mathrm{JK}$ are the perpendicular bisectors to $\mathrm{PF}, \& \mathrm{PF}_{2}$ respectively. According to the theorem EG and JK meets at line of Minor axis.

\section{Proof of the theorem}

We know that the co-ordinates of points $F_{1}$ and $F_{2}$ are $\left(\sqrt{a^{2}-b^{2}}, 0\right)$ and $\left(-\sqrt{a^{2}-b^{2}}, 0\right)$ respectively. Let, co-ordinates of point $P$ be $\left(x_{1}, y_{1}\right)$. Therefore, the

Co-ordinate of mid point of line $\mathrm{PF}_{1}$ is $\left(\frac{x_{1}+\sqrt{a^{2}-b^{2}}}{2}, \frac{y_{1}}{2}\right)$

and co-ordinate of mid point of line $\mathrm{PF}_{2}$ is $\left(\frac{x_{1}-\sqrt{a^{2}-b^{2}}}{2}, \frac{y_{1}}{2}\right)$ 
We know that the slope of the line $\mathrm{PF}_{1}$ is $\frac{y_{2}-y_{1}}{x_{2}-x_{2}}=\frac{y_{1}}{x_{1}-\sqrt{a^{2}-b^{2}}}$

Similarly, slope of the line $P F_{1}$ is $\frac{y_{2}-y_{1}}{x_{2}-x_{1}}=\frac{y_{1}}{x_{1}+\sqrt{a^{2}-b^{2}}}$

We already knew that "Two non-vertical lines are perpendicular if and only if $m_{1}$ and $m_{2}$ such that $m_{1} \times m_{2}=-1$ " where $m_{1}$ and $m_{2}$ are slopes of any two lines perpendicular to each other.

So, we know that the equation of straight line through point $\left(x_{1}, y_{1}\right)$ with slope ' $m$ ' is

$$
y-y_{1}=m\left(x-x_{1}\right)
$$

Therefore, equation of perpendicular bisector of line $\mathrm{PF}_{\mathrm{F}}$ is given by

$$
2 y y_{1}-y_{1}^{2}=2 x \sqrt{a^{2}-b^{2}}-a^{2}+b^{2}-2 x x_{1}+x_{1}^{2}
$$

Similarly, equation of perpendicular bisector of line $\mathrm{PF}_{2}$ is given by

$$
2 y y_{1}-y_{1}^{2}=-2 x \sqrt{a^{2}-b^{2}}-a^{2}+b^{2}-2 x x_{1}+x_{1}^{2}
$$

Equating equations [3] and [4], we get

$$
4 x \sqrt{a^{2}-b^{2}}=0
$$

Therefore, $x=0$ and it is nothing but $y$-axis (line of minor axis). Hence the theorem is proved.

\section{Conclusion}

The theorem is very useful for those doing research or further study in the field of ellipse or geometry, since this is also one of the important properties of an ellipse. 


\section{References}

1. M. Hazewinkel, Encyclopedia of Mathematics, Vol, Ill, Kluwer Academic Publisher, London, 1987, p. 364.

2. Louise S. Grinstein \& Sally I. Lipsey, Encyclopedia of Mathematics, Routledge Folmer, New York, 2001, p. 383.

3. E.J. Borowski \& J.M. Borwein, Collins Dictionary of Mathematics, Harper Collins Publishers, Glasgow, 1991, p. 225

4. W.Gellert. S. Gottwald, M. Hellwich, H. Köstner, H. Küstner, The VNR Concise Encyclopedia of Mathematics, Van Nostrand Reinhold, New York, 1989, p. 178.

5. http://mathworld.wolfram.com/PerpendicularBisector.html

6. E.J. Borowski \& J.M. Bonwein, Collins Dictionary of Mathematics, Harper Collins Publishers, Glasgow, 1991, p. 380. 